\title{
Designing Adaptive Coded Modulation for Optical Networks via Achievable Information Rates
}

\author{
Alex Alvarado ${ }^{1}$, David J. Ives ${ }^{2}$, and Seb J. Savory ${ }^{2}$ \\ ${ }^{1}$ Signal Processing Systems (SPS) Group, Department of Electrical Engineering \\ Eindhoven University of Technology (TU/e), 5600MB Eindhoven, The Netherlands \\ ${ }^{2}$ Department of Engineering, Electrical Division \\ University of Cambridge, CB3 OFA Cambridge, United Kingdom \\ e-mail: alex.alvarado@ieee.org
}

\begin{abstract}
Achievable information rates are discussed as a tool to analyse and design optical networks. It is shown that the maximum throughput of the network and its dependency of different network parameters can be predicted with such a tool.
\end{abstract}

Keywords: Achievable information rates, information theory, network throughput, optical networks.

\section{INTRODUCTION}

Optical networks utilise wavelength routing to transparently connect source and destination. Such networks are the backbone of the Internet and can be abstracted to a set of multiple point-to-point routes. These routes can vary considerably in length, and thus, the signal quality in optical networks-measured for example in terms of signal-to-noise ratio (SNR) — can vary considerably. While the SNR in short routes is high, long routes that connect nodes far apart experience low SNRs. This degradation is usually due to the accumulated noise introduced by amplifiers in the link as well as nonlinear distortion due to neighboring WDM channels.

Future optical networks will be spectrally efficient, and thus, they will use multilevel modulation and forward error correction (FEC). This combination is known as coded modulation (CM) [1] and its design requires the joint optimisation of the FEC and modulation format. Very often, CM receivers are implemented as a bit-wise receiver [2]. Hard or soft information on the code bits is calculated first, and then, an FEC decoder is used [3].

The most conservative CM design strategy for an optical network is to choose the transceiver to operate errorfree on the worst route [4, p. 138]. Under this design paradigm, any route reconfiguration can be accommodated through the network. However, this leads to over provisioning of resources, which has lead to an increased interest in networks with adaptive CM. In such a scenario, the modulation format and the FEC is varied to match the SNRs of the different routes. Other parameters that can be adapted include, e.g., frequency separation (flex-grid), launch power, rate, etc (see [5, Fig. 3]). The network resources can be better utilised if these degrees of freedom are jointly optimised in conjunction with the routing of the optical light path through the network.

To increase the network throughput, different approaches have been investigated in the literature. For example, variable FEC, and variable modulation, and variable symbol rate [6]-[14]. Routing and spectrum assignment for flex-grid optical networks was studied in [15]. Nonlinear effects have been taken into account using the Gaussian noise (GN) model [16]. In [17], the GN model was used to adapt the routing and wavelength assignment problem and later used in [18] to jointly optimise power, modulation format, and carrier frequencies (flex-grid). Numerical integration of the GN model was used in [19] to assess SNR and throughput optimisation via power and modulation adaptation. This methodology was also used in [20], [21] to sequentially optimise modulation format and power.

An achievable information rate (AIR) is an information-theoretic quantity that quantifies the theoretically maximum data rate that can be reliably transmitted through a channel. AIRs directly shows the dependency of the data rate on the FEC coding rate (or equivalently, FEC overhead) and the modulation format. AIRs are well understood for point-to-point links and can also be used to analyse the theoretically maximum throughput of a network. This was done for both hard-decision FEC (HD-FEC) and soft-decision FEC (SD-FEC) and different network topologies in [22]. We used the same methodology in [23] to study the effect of the amplifier noise figure on the network throughput. In this paper we review this methodology and highlight its advantages and shortcomings. While for point-to-point links and an AWGN channel AIRs are determined by the SNR, we show that for an optical network, the analysis depends on the SNR distribution of the routes.

\section{ACHIEVABLE INFORMATION RATES}

\subsection{Point-to-point Links}

The capacity of the AWGN channel under an average power constraint is $C=2 \log _{2}(1+\mathrm{SNR})$, where the pre-log factor of 2 takes into account the two polarisations. The value of $C$ represents the maximum number of information bits per symbol that can be reliably transmitted through an AWGN channel. The AWGN capacity is 
achieved when the transmitted symbols are chosen from a zero-mean Gaussian distribution. In practice, however, the modulation has $M$ discrete levels, which reduces the achievable transmission rates.

We consider a CM transmitter where a FEC encoder generates code bits. These bits are gathered in groups of $m$, and then mapped to a QAM constellation with $2^{m}=M$ constellation points. These symbols are then transmitted through a channel. At the receivers, two structures are considered. The first one is based on a HDFEC decoder. In this case, the noisy symbols are first converted into bits ( $m$ bits per received symbol). These bits are passed to an HD-FEC decoder. In the second structure, the noisy symbols are converted into "soft" bits (usually log-likelihood ratios), which are then passed to an SD-FEC decoder.

For HD-FEC, Shannon's coding theorem states that error-free transmission is possible if

$$
R_{\mathrm{c}} \leq 1-H_{\mathrm{b}}(\mathrm{BER})=1+\mathrm{BER} \cdot \log _{2} \mathrm{BER}+(1-\mathrm{BER}) \cdot \log _{2}(1-\mathrm{BER}),
$$

where $R_{\mathrm{c}}$ is the rate of the FEC encoder, $H_{\mathrm{b}}(\cdot)$ is the binary entropy function, and BER is the average pre-FEC BER (across $m$ bit positions). The AIR is therefore $m\left(1-H_{\mathrm{b}}(\mathrm{BER})\right)$ (in bit per symbol) and it represents the maximum number of bits that can be reliably transmitted in each symbol.

The values of the AIRs $m\left(1-H_{\mathrm{b}}(\mathrm{BER})\right)$ for $M=4,16, \ldots, 1024$ are shown in Fig. 1 (left). The results are obtained for an AWGN channel and 2 polarisations. These AIRs cross each other for different values of $M$ (see the asterisks in Fig. 1), and thus, the theoretically optimal choice of $R_{\mathrm{c}}$ and $M$ is not straightforward. For HD-FEC we consider here, QPSK should be used for SNRs below $5.8 \mathrm{~dB}, 16 \mathrm{QAM}$ for $5.8 \leq \mathrm{SNR} \leq 14 \mathrm{~dB}$, etc. The corresponding FEC OHs are shown in Fig. 1 (right). This figure also shows the optimum minimum and maximum $\mathrm{OH}$ values for each modulation format.
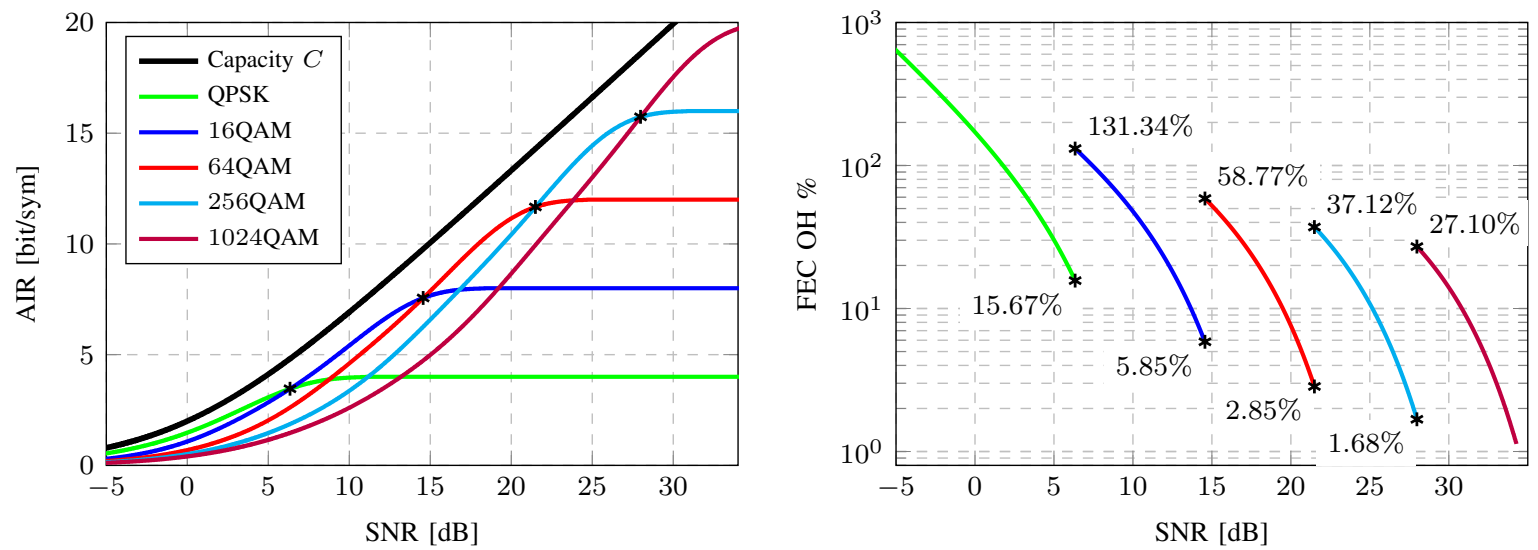

Figure 1. AIRs with HD-FEC (left) and corresponding OHs (right). The black asterisks show the SNR values where the modulation size should be changed. The channel capacity $C$ is shown for comparison.

\subsection{Optical Networks}

The performance metric under consideration is the total network throughput, which we denote by $\Theta$. For a precise definition of $\Theta$, we refer the reader to [22, Sec. III]. The network throughput represents the total traffic transported by the network that satisfies a given traffic demand.

To solve the network throughput problem, the routing and wavelength assignment problem was solved numerically as an integer linear programming (ILP) problem as described in [21, Section 4.1]. In particular, we maximised the network throughput $\Theta$ under a uniform traffic demand assuming the transceivers achieve the channel capacity $C$, and for a fixed channel separation $(50 \mathrm{GHz})$. All other system parameters are given in [22, Table I]. The channel capacity $C$ is pre-calculated based on the route SNR, which is in turn calculated using the GN model assuming a uniform launch power that maximises the SNR of the worst case central wavelength channel (see more details in [22, Sec. II-C]).

The ILP solution provides the number of active light paths (i.e., number of transceivers) and their routes between each node pair. From this, the total number of active light paths $T$ in the network is obtained. This solution also provides the SNR of each active light path. The obtained results are shown in Fig. 2 for three network topologies: national (Deutsche Telekom Germany (DTG)), continental (NSF topology), and transcontinental (Google B4 (GB4)). These networks are described in detail in [22, Sec. II-B]. The vertical bars show the number of transceivers that need to be installed to maximise the network throughput. The total number of transceivers for the DTG, NSF, and DTG networks are 1230, 1094, and 570, respectively.

The vertical bars in Fig. 2 can be interpreted as the SNR distribution across the network. By comparing these distributions, it is clear that the average SNRs across the network decreases as the size of the network increases. 

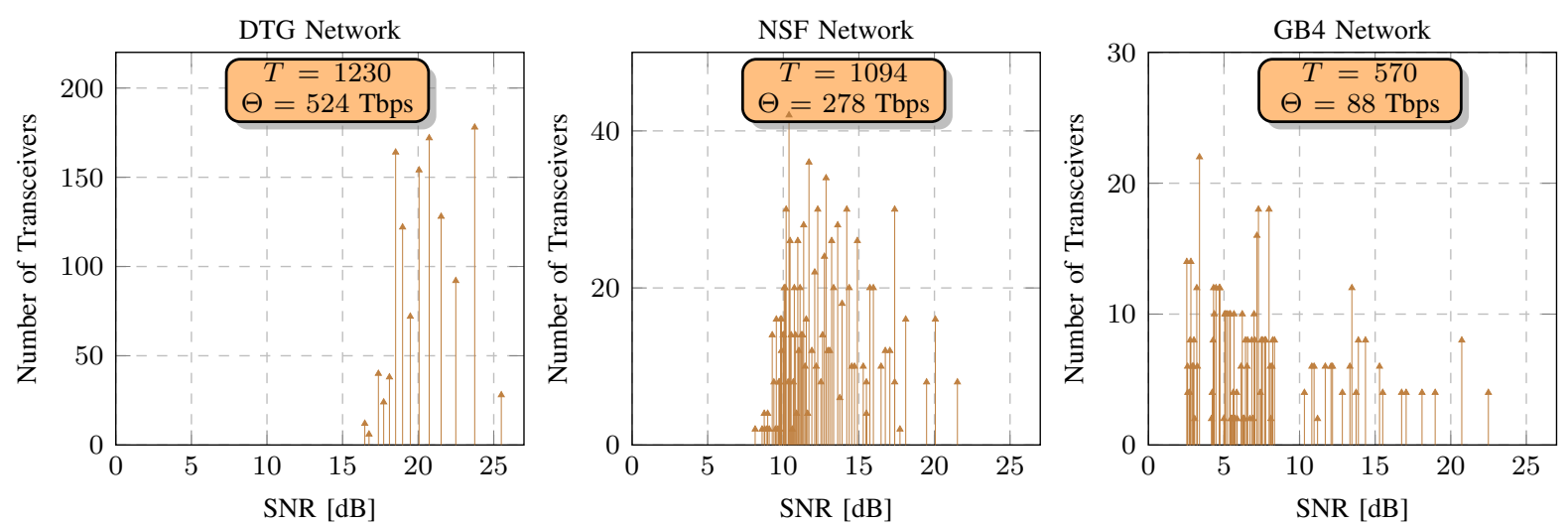

Figure 2. Number of transceivers (vertical lines with triangles at the top) for all SNR values for the three network topologies assuming all transceivers achieve the capacity $C$. Network throughputs $\Theta$ are also shown.

This is due to the presence of long links in continental and transcontinental networks (NSF and GB4). The SNR distributions in Fig. 2 also show that the spread of the SNR values is much larger for large networks. While for the DTG network, the variation in SNR is about $10 \mathrm{~dB}$, for the GB4 network, this variation is about $20 \mathrm{~dB}$.

Once the SNR values for the active light paths are found, the maximum network throughput can be calculated using AIRs. In particular, the SNRs of the light paths shown in Fig. 2 are first grouped for each source destination pair. Then, the SNRs are "mapped" to throughputs AIR curves. In the ideal case when transceivers achieve the channel capacity, the resulting throughputs are 524, 278, and 88 Tbps (see Fig. 2). For the more realistic case of discrete constellations, this mapping approach can still be used, via the results in Fig. 1. This is shown schematically in Fig. 3 (left) for the DTG network. These idealised results show that the transceivers should only use 64QAM and 256QAM. The results in Fig. 3 (right) show similar results for SD-FEC. In this case, AIRs similar to those in Fig. 1 can be obtained based on the generalised mutual information [2], [3]. The results in Fig. 3 (right) show how most of the transceivers should use 256QAM and 1024QAM (for the short routes with high SNRs).
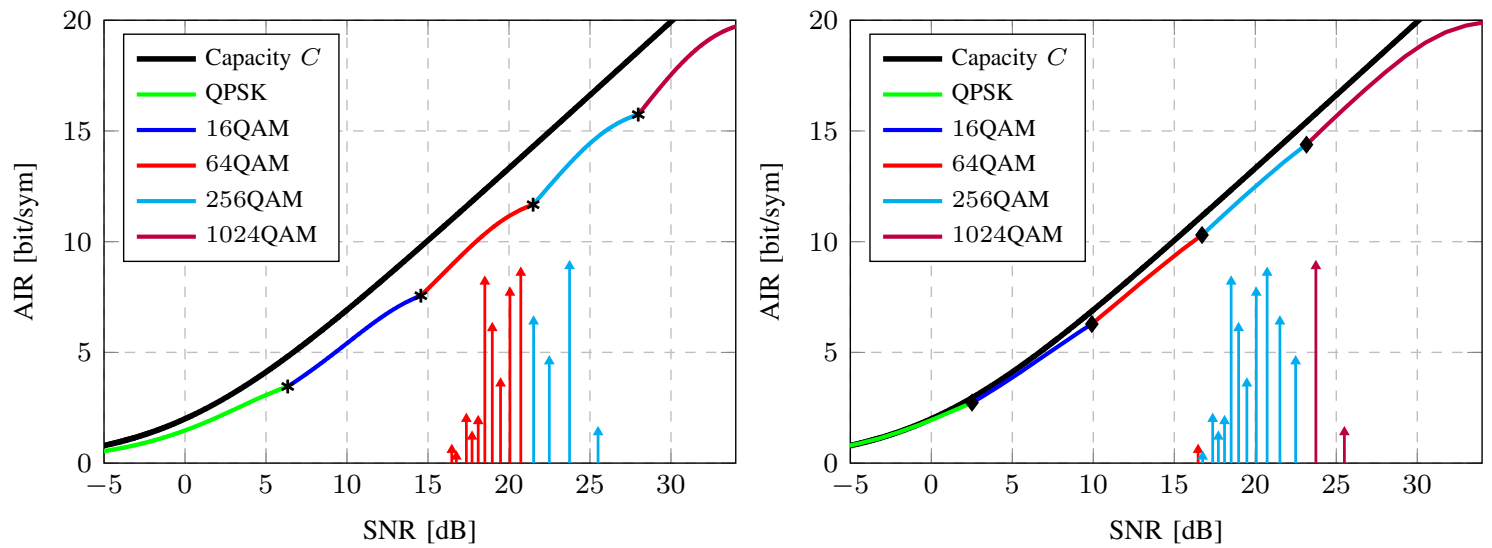

Figure 3. Optimum modulation format for HD-FEC (left) and SD-FEC (right) as well as the SNR distribution for the DTG network.

\subsection{Applications: Code Rates and Amplifier Noise Figure}

One of the key components in an optical network are the EDFAs, which are characterised by their noise figure (NF). This NF varies between $3 \mathrm{~dB}$ (the theoretical minimum) and $5-7 \mathrm{~dB}$ (for most commercially available amplifiers). The results in the previous section were obtained for a NF of $5 \mathrm{~dB}$. Here, we use the methodology described above to quantify the dependency of the network throughout on the amplifier NF.

The obtained results are shown in Fig. 4. For $\mathrm{NF}=5 \mathrm{~dB}$, the highlighted throughputs are those obtained in Fig. 3 and show the potential gains offered by SD-FEC over HD-FEC. These result show that the gains offered by SD-FEC are much more important in large networks. For example, the relative throughput gains for the DTG network are, on average, 13\%; for the GB4 network these gains increase to $28 \%$. The results in Fig. 4 also show the tradeoff between SD-FEC and amplifier noise figure: for all three networks, a given throughput obtained with HD-FEC can be maintained if SD-FEC is implemented and the NF is increased by $3 \mathrm{~dB}$. 


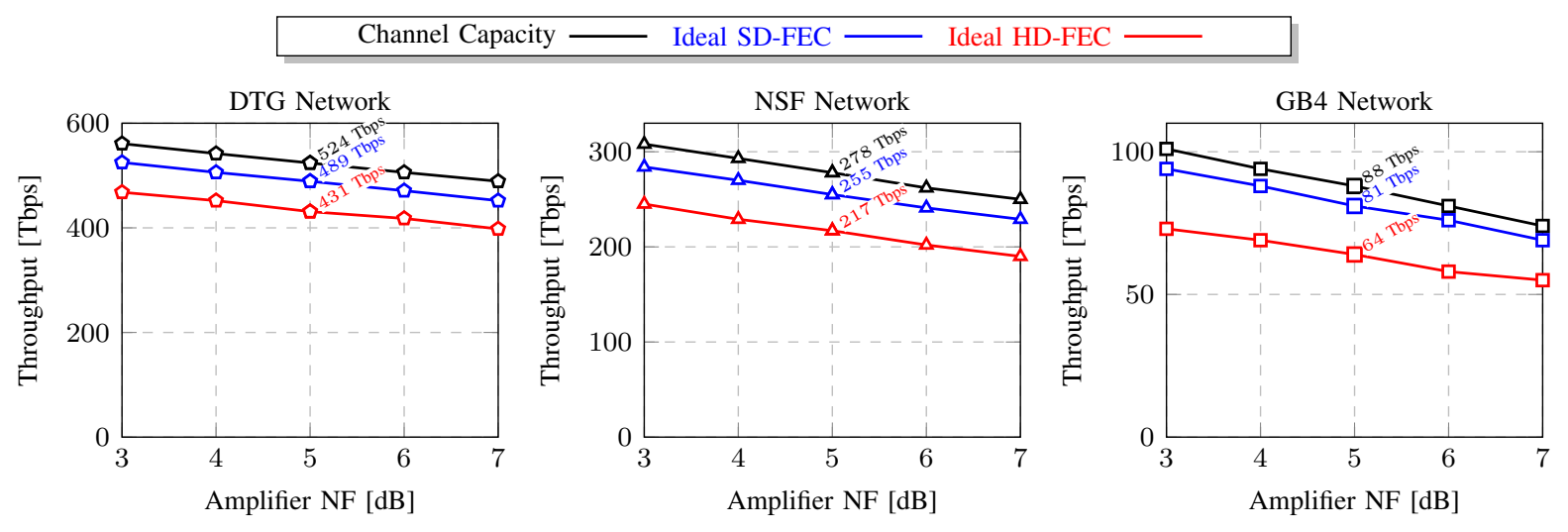

Figure 4. Network throughputs for three different network topologies as a function of the amplifier NF: transceivers achieving the channel capacity (black lines), SD-FEC (blue lines), and HD-FEC (red lines).

\section{CONCLUSIONS}

In this paper we discussed the use of information-theoretic metrics (achievable information rates) in the design of optical networks. It was shown that achievable information rates are a powerful design tool for networks that use forward error correction and multilevel modulation. The main focus of this paper was on uniform traffic and idealised forward error correction. Future work includes analysis of dynamic traffic scenarios and practical codes. We believe the methodology discussed in this paper can be used in such cases.

\section{REFERENCES}

[1] G. Ungerboeck, "Channel coding with multilevel/phase signals," IEEE Trans. Inf. Theory, vol. 28, no. 1, pp. 55-67, Jan. 1982.

[2] L. Szczecinski and A. Alvarado, Bit-Interleaved Coded Modulation: Fundamentals, Analysis and Design. Chichester, UK: John Wiley \& Sons, 2015.

[3] A. Alvarado and E. Agrell, "Four-dimensional coded modulation with bit-wise decoders for future optical communications," J. Lightw. Technol., vol. 33, no. 10, pp. 1993-2003, May 2015.

[4] M. Jinno, B. Kozicki, H. Takara et al., "Distance-adaptive spectrum resource allocation in spectrum-sliced elastic optical path network," IEEE Commun. Mag., vol. 48, no. 8, pp. 138-145, Aug. 2010.

[5] S. Gringeri, N. Bitar, and T. J. Xia, "Extending software defined network principles to include optical transport," IEEE Commun. Mag., vol. 52, no. 3, pp. 32-40, Mar. 2013.

[6] A. Nag, M. Tornatore, and M. Mukherjee, "Optical network design with mixed line rates and multiple modulation formats," J. Lightw. Technol., vol. 28, no. 4, pp. 466-475, Feb. 2010.

[7] G.-H. Gho and J. M. Kahn, "Rate-adaptive modulation and low-density parity-check coding for optical fiber transmission systems," J. Opt. Commun. Netw., vol. 4, no. 10, pp. 760-768, Oct. 2012.

[8] D. A. A. Mello, A. N. Barreto, T. C. de Lima et al., "Optical networking with variable-code-rate transceivers," J. Lightw. Technol., vol. 32, no. 2, pp. 257-266, Jan. 2014.

[9] R. Schmogrow, D. Hillerkuss, M. Dreschmann et al., "Real-time software-defined multiformat transmitter generating 64QAM at 28 GBd," IEEE Photon. Technol. Lett., vol. 22, no. 21, pp. 1601-1603, Nov. 2010.

[10] H. Y. Choi, T. Tsuritani, and I. Morita, "BER-adaptive flexible-format transmitter for elastic optical networks," Opt. Express, vol. 20, no. 17 , pp. $18652-18658$, Aug. 2012

[11] B. T. Teipen, M. H. Eiselt, K. Grobe et al., "Adaptive data rates for flexible transceivers in optical networks," Journal of Networks, vol. 17, no. 5, pp. 776-782, May 2012.

[12] H. Y. Choi, L. Liu, T. Tsuritani et al., "Demonstration of BER-Adaptive WSON employing flexible transmitter/receiver with an extended openflow-based control plane," IEEE Photon. Technol. Lett., vol. 25, no. 2, pp. 119-121, Jan. 2013.

[13] N. Sambo, G. Meloni, F. Cugini et al., "Routing, code, and spectrum assignment, subcarrier spacing, and filter configuration in elastic optical networks," Journal of Optical Communications and Networking, vol. 11, no. 7, pp. B93-B100, Nov. 2015, (Invited Paper).

[14] Y. Li, H. Dai, G. Shen et al., "Adaptive FEC-based lightpath routing and wavelength assignment in WDM optical networks," Optical Switching and Networking, vol. 14, no. 3, pp. 241-249, Aug. 2014.

[15] K. Christodoulopoulos, I. Tomkos, and E. Varvarigos, "Elastic bandwidth allocation in flexible OFDM-Based optical networks," $J$. Lightw. Technol., vol. 29, no. 9, pp. 1354-1366, May 2011.

[16] P. Poggiolini, G. Bosco, A. Carena et al., "The GN-model of fiber non-linear propagation and its applications," J. Lightw. Technol., vol. 32, no. 4, pp. 694-721, Oct. 2014 (Invited Tutorial).

[17] J. Zhao, H. Wymeersch, and E. Agrell, "Nonlinear impairment aware resource allocation in elastic optical networks," in Proc. OFC, Los Angeles, CA, Mar. 2015.

[18] L. Yan, E. Agrell, and H. Wymeersch, "Link-level resource allocation for flexible-grid nonlinear fiber-optic communication systems," IEEE Photon. Technol. Lett., vol. 27, no. 12, pp. 1250-1253, June 2015.

[19] D. J. Ives, P. Bayvel, and S. J. Savory, "Adapting transmitter power and modulation format to improve optical network performance utilizing the Gaussian noise model of nonlinear impairments," J. Lightw. Technol., vol. 32, no. 21, pp. 4087-4096, Nov. 2014.

[20] — - "Physical layer transmitter and routing optimization to maximize the traffic throughput...," in Proc. Int. Conf. on Optical Network Design and Modeling, Stockholm, Sweden, May 2014

[21] _ - "Routing, modulation, spectrum and launch power assignment to maximize the traffic throughput ...," Photonic Network Communications, vol. 29, no. 3, pp. 244-256, Mar. 2015.

[22] A. Alvarado, D. J. Ives, S. Savory et al., "On the impact of optimal modulation and FEC overhead on future optical networks," $J$. Lightw. Technol., vol. 34, no. 9, pp. 2339-2352, May 2016.

[23] _ , "Impact of amplifier noise figure on network throughput," in Proc. Optical Fiber Communication Conference (OFC), Anaheim, CA, Mar. 2016. 